\title{
Technical quality of root canal treatment in Taiwan
}

\author{
L.-H. Chueh${ }^{1}$, S. -C. Chen ${ }^{2}$, C.-M. Lee ${ }^{3}$, Y.-Y. Hsu' ${ }^{1}$, S.-F. Pai ${ }^{4}$, M.-L. Kuo ${ }^{5}$, C.-S. Chen ${ }^{6}$, \\ B.-R. Duh ${ }^{7}$, S. -F. Yang ${ }^{4}$, Y.-L. Tung ${ }^{8}$ \& C. K. Hsiao ${ }^{8}$ \\ ${ }^{1}$ Elite Dental Clinic, ${ }^{2}$ Poling Dental Clinic, ${ }^{3}$ Shin Kong Wu Ho Su Memorial Hospital, ${ }^{4}$ Endodontic Section, Dental Department, \\ Veterans General Hospital-Taipei and School of Dentistry, NationalYang-Ming University, ${ }^{5}$ Landmark Dental Clinic, ${ }^{6}$ Department of \\ Endodontics, Cathay General Hospital, ${ }^{7}$ Mackay Memorial Hospital, ${ }^{8}$ Division of Biostatistics, Graduate Institute of Epidemiology, \\ College of Public Health, National Taiwan University,Taipei,Taiwan
}

\begin{abstract}
Chueh L-H, Chen S-C, Lee C-M, Hsu Y-Y, Pai S-F, Kuo M-L, Chen C-S, Duh B-R, Yang S-F, Tung Y-L, Hsiao CK. Technical quality of root canal treatment in Taiwan. International Endodontic Journal, 36, 416-422, 2003.
\end{abstract}

Aim To evaluate the current technical quality of root canal treatment (RCT) in Taiwan.

Methodology A total of 1085 RCT cases, randomly selected from a large sample and representative of the Taiwanese population from April to September 2000, were evaluated by eight endodontic specialists. The qualitative evaluation of RCT cases was based on two variables: length of the root filling and density of the obturation. A root canal with both adequate filling length (the apical termination of the root filling within $2 \mathrm{~mm}$ of the radiographic apex) and complete obturation (no lateral or apical canal lumen visible in the apical one-third of the root canal) was defined as having good-quality endodontic work (GQEW). A tooth was defined as having a GQEW when all its canals were categorized as GQEW.
Results From a total of 1867 root canals, overfilling occurred in 235 (12.6\%), adequate filling length in $1152(61.7 \%)$, underfilling in $466(25.0 \%)$ and no filling in $12(0.6 \%)$. Of the 1867 root canals, 710 $(38.0 \%)$ demonstrated complete obturation and 1157 $(62 \%)$ demonstrated incomplete obturation. GQEW was found in $650(34.8 \%)$ root canals and 329 (30.3\%) teeth. The percentage of teeth with GQEW in hospital cases $(38.1 \%)$ was significantly greater $(P<0.001)$ than that in private clinic cases $(24.3 \%)$. In addition, the frequency of teeth with GQEW in the anterior teeth $(40.4 \%)$ or in the premolars $(33 \%)$ was significantly greater $(P<0.001)$ than that in the molars $(18.4 \%)$.

Conclusions Approximately $70 \%$ of the teeth receiving RCT in Taiwan were either of inadequate filling length or sealing density.

Keywords: root canal treatment, Taiwan, technical quality.

Received 23 September 2002; accepted 18 February 2003

\section{Introduction}

Endodontic treatment is an essential part of comprehensive quality dental care. Controlled studies have shown that root canal treatment (RCT) brings high success rates of more than 90\% (Sjögren et al. 1990, Eriksen 1991, Friedman 1998). Most of these studies reported data from endodontic specialists and university clinics. These data may mislead in estimating the success rate of endodontic treatment in general practice that approximates to

Correspondence: Chuhsing Kate Hsiao, Division of Biostatistics, Graduate Institute of Epidemiology, College of Public Health, National Taiwan University, No. 1, Section 1, Jen-Ai Road, 15th Floor, Room 1542, Taipei, Taiwan (Fax: +8862 23418562; e-mail: ckhsiao@ha.mc.ntu.edu.tw).
65-75\% (Eriksen 1991, Friedman 1998).This discrepancy in success rate may reflect a difference in the technical quality of the endodontic treatment performed.

Epidemiological surveys indicate a markedly lower frequency of satisfactory results of root canal filling ranging from 14 to $65 \%$ (Petersson et al. 1986, Eckerbom et al. 1987; 1989, Ödesjö et al. 1990, Imfeld 1991, De Cleen et al. 1993, Buckley \& Spångberg 1995, Saunders et al. 1997, Weiger et al. 1997, Marques et al. 1998, Sidaravicius et al. 1999, De Moor et al. 2000, Kirkevang et al. 2000; 2001, Boucher et al. 2002). In order to evaluate the endodontic status of the population, numerous studies have focused on the technical quality of RCT. This is of interest because it is well known that the quality of the root canal filling is judged to be an important factor for the 
prognosis of the RCT (Petersson et al.1986, Eckerbom etal. 1989, Ödesjö et al. 1990, Imfeld 1991, De Cleen et al. 1993, Buckley \& Spångberg 1995, Saunders et al. 1997, Weiger et al. 1997, De Moor et al. 2000, Kirkevang et al. 2000, Boucher et al. 2002).

In Taiwan, the national healthcare policy was implemented in March 1995. Because the service is almost free, the utilization of dental service has risen exponentially. In order to avoid abuse and fraud when claiming an RCT fee, the providers need to submit the RCT cases randomly selected by the Bureau of National Health Insurance (BNHI) in Taiwan for peer reviewing with thorough dental records including pre- and post-treatment periapical radiographs. With these invaluable data at BNHI, it will be possible to study the technical quality of RCT in the whole Taiwanese population. In October 2000, BNHI in Taiwan announced an incentive programme for RCT. Under the programme, the RCT cases that fulfilled certain requirements, i.e. rubber dam placement and adequate root filling length with a complete obturation, could get better payment from BNHI. In order to compare the quality status of RCT before and after the announcement of this incentive programme, the aim of this study was to assess the current technical quality of RCT in the whole Taiwanese population. A future investigation that is designed to disclose whether there is any improvement in the technical quality of RCT under the stimulation of this incentive programme will be performed 3 years later.

\section{Materials and methods}

\section{Selection of cases}

The design of random sampling stratified by the geographical area and the hospital type was used. The sample size (n) was estimated to be 1200 so that the standard error (SE) of the estimate $\hat{p}$ could be controlled to be less than 0.05 via the formula $(s e)^{2}=\hat{p}(1-\hat{p}) / n$. According to the regulations of $\mathrm{BNHI}, 5 \%$ of the RCT cases performed by the dentists in hospitals and $10 \%$ of the RCT cases performed by the dentists in private clinics should be randomly selected and submitted to BNHI for peer reviewing. In this study, one to three cases were chosen from each randomly selected hospital or private clinic, according to the list of random numbers that was prepared by the statisticians. A total of 1183 cases ( 523 from hospitals and 660 from private clinics) were randomly selected from approximately 150000 RCT cases submitted to BNHI for peer reviewing from April to September 2000. These 1183 cases were collected from randomly selected 187 hospitals and 831 private clinics within Taiwan. Of the 1183 cases, 383 (32.4\%) were anterior teeth (incisors and canines), 361 (30.5\%) were premolars and 439 (37.1\%) were molars (Table 1). The gender of the patients was not documented in 29 patients. Of the remaining 1154 patients, 645 (55.9\%) were females and $509(44.1 \%)$ were males. All the patients were adults (age $\geqq 20$ years). The mean number of periapical radiographs submitted was 2.30 per case. Ninety-eight cases $(8.3 \%, 48$ from hospitals and 50 from private clinics), whose radiographs were inadequate for interpretation as a result of poor radiographic technique or processing, were excluded. The remaining 1085 teeth (475 from hospitals and 610 from private clinics), whose radiographs were readable, constituted the basic material for evaluation in this study. These 1085 teeth included 361 anterior teeth, 339 premolars and 385 molars (Table 1).

\section{Evaluation of the quality of root canal treatment}

A group of eight endodontists were trained and calibrated to evaluate the quality of the collected endodontic cases. An evaluation form was designed to record the information gathered from the submitted charts and radiographs. Each set of radiographs from one case was interpreted by one of the eight endodontists. The viewing conditions were standardized, using an illuminated viewing box with fixed light. The technical quality of the root canal fillings was evaluated and classified. The recorded data related to this report included tooth type,

Table 1 Tooth types of 1183 teeth from either hospitals or private clinics

\begin{tabular}{|c|c|c|c|c|c|c|}
\hline \multirow[b]{2}{*}{ Tooth types } & \multicolumn{2}{|l|}{ Hospitals } & \multicolumn{2}{|c|}{ Private clinics } & \multicolumn{2}{|l|}{ Total } \\
\hline & Readable & Unreadable & Readable & Unreadable & Number & $\%$ \\
\hline Anterior teeth & 179 & 11 & 182 & 11 & 383 & 32.4 \\
\hline Premolars & 149 & 7 & 190 & 15 & 361 & 30.5 \\
\hline Molars & 147 & 30 & 238 & 24 & 439 & 37.1 \\
\hline Total & 475 & 48 & 610 & 50 & 1183 & 100 \\
\hline
\end{tabular}


filling material, root canal filling length and density of root canal filling.

The root canal filling material of each case was assessed by radiographs and dental charts. Gutta-percha was judged as the filling material, when gutta-percha core material could be identified from the radiograph or when gutta-percha was recorded in the dental chart as the filling material. When no recording in the notes could be found but the radiopacity and homogeneity were similar to gutta-percha, it was classified as guttapercha-like material. When silver point or paste was used as the filling material, it was categorized as 'others'.

The qualitative evaluation of RCT cases was based on two variables: length of the root filling and density of the obturation in the apical one-third of the root canal. Multicanal teeth with superimposed canal fillings recorded on the dental chart were assessed according to the same radiographic image for both canals. By this definition, overfilling was defined as seeing filling material beyond the radiographic apex. Root canal filling was judged as adequate when the apical termination of the root filling was within $2 \mathrm{~mm}$ of the radiographic apex. Furthermore, root canal filling was classified as underfilled when the root filling ended more than $2 \mathrm{~mm}$ from the radiographic apex, and as not filled when no filling material was visible in the root canal. Density of the obturation at the apical one-third of the root canal was classified as complete when no lateral or apical canal lumen was visible and as incomplete when there was a canal lumen visible laterally or apically. A root canal with both adequate filling length and complete obturation in the apical one-third of the root canal was defined as having a good-quality endodontic work (GQEW). A treated tooth was defined as having a GQEW when all its canals had a GQEW.

\section{Statistical evaluation}

Interexaminer variability was studied through independent examination of 20 randomly selected cases of radiographs from the same source prior to the investigation. Interexaminer agreement was measured by Cohen's kappa (к) values (Cohen 1960, Valachovic et al. 1986). The $\kappa$-statistic was also used to assess the reproducibility within each rater (intra-examiner). To evaluate the intra-examiner reproducibility, 20 randomly selected cases were evaluated by the eight endodontists; the same cases were again re-examined 4 weeks later. An alternative measurement, the percent agreement (or proportion of agreement), was also used to measure intra-examiner variability (Hunt 1986, Valachovic et al. 1986).
Sample means and their standard errors were used to describe every item listed on the evaluation form. The Chi-square statistic was used to test the null hypothesis which states that the mean proportions of root canals or teeth with GQEW from the populations are identical. Once the comparison amongst these groups was statistically significant $(P$-value $<0.05)$, the Bonferroni multiple comparison procedure was then used to find out whether all the mean proportions or only some of them were different (Glantz 1997).

\section{Results}

The $\kappa$-value for interexaminer variability was 0.80 for 'filling material', 0.71 for 'length of root filling' and 0.52 for 'obturation of root canal'. The $\kappa$-values for intraexaminer reproducibility of the eight endodontic specialists ranged from 0.64 to 1 for 'length of root filling' and from 0.19 to 0.87 for 'obturation of root canal'. The values of percent agreement for measuring the intraexaminer variability were all greater than 0.75 .

Root canal filling materials in 1085 teeth with RCTare shown in Table 2. Of these 1085 teeth, gutta-percha was the main filling material in 735 (67.7\%), guttapercha-like material in $293(27.0 \%)$ and other materials in $57(5.3 \%)$ teeth (Table 2).

Detailed information about root filling length in 1867 root canals from 1085 teeth with RCT is shown in Table 3. Of the 1867 root canals, 788 were from hospitals and

Table 2 The numbers and percentages (in parentheses) of teeth with different filling materials in 1085 teeth with root canal treatment

\begin{tabular}{lclc}
\hline Filling materials & Hospitals & Private clinics & Total \\
\hline Gutta-percha & $380(80.0)$ & $355(58.2)$ & $735(67.7)$ \\
Gutta-percha like & $79(16.6)$ & $214(35.1)$ & $293(27.0)$ \\
Others & $16(3.4)$ & $41(6.7)$ & $57(5.3)$ \\
Total & $475(100)$ & $610(100)$ & $1085(100)$ \\
\hline
\end{tabular}

Table 3 Numbers and percentages (in parentheses) of root canals with different conditions of filling length in 1867 root canals from 1085 teeth with root canal treatment

\begin{tabular}{lccc}
\hline Filling length & Hospitals & Private clinics & Total \\
\hline Overfilling & $90(11.4)$ & $145(13.4)$ & $235(12.6)$ \\
Adequate filling length & $527(66.9)$ & $625(57.9)$ & $1152(61.7)$ \\
Underfilling & $164(20.8)$ & $302(28.0)$ & $466(25.0)$ \\
No filling & $7(0.9)$ & $5(0.5)$ & $12(0.6)$ \\
Data unavailable & $0(0)$ & $2(0.2)$ & $2(0.1)$ \\
Total & $788(100)$ & $1079(100)$ & $1867(100)$ \\
\hline
\end{tabular}


Table 4 Numbers and percentages (in parentheses) of root canals with complete or incomplete obturation of the apical one third of the canal in 1867 root canals from 1085 teeth

\begin{tabular}{llcr}
\hline $\begin{array}{l}\text { Density of root } \\
\text { canal obturation }\end{array}$ & Hospitals & Private clinics & \multicolumn{1}{l}{ Total } \\
\hline Complete & $369(46.8)$ & $341(31.6)$ & $710(38.0)$ \\
Incomplete & $419(53.2)$ & $738(68.4)$ & $1157(62.0)$ \\
Total & $788(100)$ & $1079(100)$ & $1867(100)$ \\
\hline
\end{tabular}

1079 from private clinics. Data of root filling length were not available in the two root canals from private clinics. The remaining 1865 root canals showed overfilling in 235 (12.6\%), adequate filling length in $1152(61.7 \%)$, underfilling in $466(25.0 \%)$ and no filling in $12(0.6 \%)$ (Table 3).

Density of root canal obturation in 1867 root canals from 1085 teeth with RCT is shown in Table 4. Of 1867 root canals, 710 (38\%) demonstrated complete obturation and $1157(62 \%)$ showed incomplete obturation (Table 4).

GQEW, defined as having both adequate filling length and complete obturation, was found in 650 (34.8\%) root canals, which included 339 from hospitals and 311 from private clinics (Table 5). After adjustment as a result of stratified sampling, the weighted average of root canals with GQEW was $30 \%$ with an SE of 0.01 . The percentage of root canals with GQEW in hospital cases $(43.0 \%)$ was significantly greater than that $(28.8 \%)$ in private clinic cases $(P<0.001)$. Of the 650 root canals with GQEW, 146 were from anterior teeth, 154 from premolars and 350 from molars. A significant difference in the frequency of root canals with GQEW was found amongst the anterior teeth $(40.4 \%)$, premolars $(34.3 \%)$ and molars $(33.1 \%)(P<0.05)$. In addition, through multiple comparisons, the percentage of root canals with GQEW in the anterior teeth was significantly greater than that in the molars $(P<0.05)$. However, there was no significant difference in the frequency of root canals with GQEW between premolars and anterior teeth or molars (Table 5).

Detailed information about the percentage of teeth with GQEW according to tooth types is shown in Table 6 . GQEW in a tooth, i.e. a tooth with its single canal or all multiple canals showing a GQEW, was found in 329 $(30.3 \%)$ teeth. After adjustment as a result of stratified sampling, the weighted average of teeth with GQEW was $25 \%$ with an SE of 0.02 . The percentage of teeth with GQEW in hospital cases (38.1\%) was significantly greater than that $(24.3 \%)$ in private clinic cases $(P<0.001)$. The frequency of teeth with GQEW in anterior teeth $(40.4 \%)$ or in premolars $(33.0 \%)$ was significantly higher
Table 5 Percentage of root canals with good-quality endodontic work (GQEW) according to tooth types

\begin{tabular}{lccl}
\hline \multirow{2}{*}{ Tooth types } & \multicolumn{3}{l}{ Root canals with GQEW/total (percentage) } \\
\cline { 2 - 4 } & Hospitals & Private clinics & Overall \\
\hline Anterior teeth & $83 / 179(46.4)$ & $63 / 182(34.6)$ & $146 / 361(40.4)^{\mathrm{a}, \mathrm{b}}$ \\
Premolars & $83 / 204(40.7)$ & $71 / 245(29.0)$ & $154 / 449(34.3)^{\mathrm{a}}$ \\
Molars & $173 / 405(42.7)$ & $177 / 652(27.1)$ & $350 / 1057(33.1)^{\mathrm{a}}$ \\
Total & $339 / 788(43.0)^{\mathrm{c}}$ & $311 / 1079(28.8)$ & $650 / 1867(34.8)$ \\
\hline
\end{tabular}

${ }^{a}$ Comparison of the frequency of root canals with GQEW amongst three tooth types; $P<0.05$.

${ }^{\mathrm{b}}$ Multiple comparison of the frequency of root canals with GQEW between anterior and molar teeth; $P<0.05$.

${ }^{\mathrm{c}}$ Comparison of the frequency of root canals with GQEW between hospital and private clinic cases; $P<0.001$.

Table 6 Percentage of teeth with good-quality endodontic work (GQEW) according to tooth types

\begin{tabular}{llcl}
\hline \multirow{2}{*}{ Tooth types } & \multicolumn{3}{l}{ Teeth with GQEW/total (percentage) } \\
\cline { 2 - 4 } & Hospitals & Private clinics & Overall \\
\hline Anterior teeth & $83 / 179(46.4)^{\mathrm{a}}$ & $63 / 182(34.6)^{\mathrm{a}}$ & $146 / 361(40.4)^{\mathrm{a}}$ \\
Premolars & $60 / 149(40.3)^{\mathrm{b}}$ & $52 / 190(27.4)^{\mathrm{c}}$ & $112 / 339(33.0)^{\mathrm{c}}$ \\
Molars & $38 / 147(25.9)$ & $33 / 238(13.9)$ & $71 / 385(18.4)$ \\
Total & $181 / 475(38.1)^{\mathrm{d}}$ & $148 / 610(24.3)$ & $329 / 1085(30.3)$ \\
\hline
\end{tabular}

${ }^{\text {a } M u l t i p l e ~ c o m p a r i s o n ~ o f ~ t h e ~ f r e q u e n c y ~ o f ~ t e e t h ~ w i t h ~ G Q E W ~ b e t w e e n ~}$ anterior and molar teeth; $P<0.001$.

${ }^{\mathrm{b}}$ Multiple comparison of the frequency of teeth with GQEW between premolar and molar teeth; $P<0.05$.

${ }^{\mathrm{c}}$ Multiple comparison of the frequency of teeth with GQEW between premolar and molar teeth; $P<0.001$.

${ }^{\mathrm{d}}$ Comparison of the frequency of teeth with GQEW between hospital and private clinic cases; $P<0.001$.

than that $(18.4 \%)$ in molars $(P<0.001)$. However, there was no significant difference in the frequency of teeth with GQEW between the anterior teeth and the premolars. Similar trends were found when comparing the frequency of teeth with GQEW in both hospital and private clinic cases according to the tooth types (Table 6).

\section{Discussion}

This paper was the first investigation on current technical quality of RCT in Taiwan using the whole Taiwanese population as the sampling source. Different from other published data, RCT cases that had been completed in recent months were evaluated. Each one of the cases was subjected to sampling, regardless of success or failure. Conversely, all the other epidemiological studies had evaluated the technical quality of root canal treatments without the knowledge of the date of treatment. 
In addition, some of the failed cases might have been retreated or extracted when the symptoms or signs developed after the initial treatment. Such a second intervention on previously treated cases could affect the results of these studies. Because the cases in our study were randomly selected from a nation-wide large sample completed within a period of 6 months, the results reflected the current status of technical quality of RCT in the whole Taiwanese population.

In the present study, periapical lesions associated with teeth with RCT were not assessed because the periapical radiographs were submitted from different institutions without a standardized radiographic technique. A total of $8.3 \%$ of the periapical radiographs were classified as unreadable as a result of poor radiographic technique or processing. In some of these excluded cases, the root apex was not visible in the radiographs; in others, the images were too vague to be interpreted. Furthermore, even in some of the readable samples, the apical area of the treated tooth was not included in the periapical radiographs because inclusion of the apical area of the treated tooth was not required by BNHI. This was the reason why, in this study, the periapical lesions of the teeth with RCT were not assessed.

The $\kappa$-values of 0.80 and 0.71 indicate excellent agreement in the ratings of 'filling material' and 'length of root filling' amongst the eight endodontists. The $\kappa$-value of 0.52 for 'obturation of root canal' represents fair agreement. This may be because of the fact that there are only two choices, 'complete' or 'incomplete' for the endodontists to select for the density of root canal obturation in this study. When observations are binary, the interrater variability may not be described well using $\kappa$-statistic. To measure the intraexaminer variability, the values of the proportion of agreement were all greater than 0.75 , indicating high consistency of each endodontist. When $\kappa$-value was used for measurement, it again gave evidence of the consistent standard of each rater in this study. Data of inter- and intraexaminer variability were comparable to those reported by others. Petersson et al. (1986) reported an interexaminer agreement of $68 \%$ for three examiners and an intraexaminer reproducibility of 76,79 and $93 \%$, respectively, for the same three examiners, regarding the evaluation of technical quality of the root fillings. Furthermore, Kirkevang et al. (2000) reported an intra-observer agreement $\kappa$-value of 0.74 for 'length of root filling' and of 0.61 for 'obturation of root canal.

Amongst 1867 root canals examined, adequate filling length was observed in $61.7 \%$ and complete obturation in $38 \%$ of the root canals. According to the regulations of BNHI, filling length is more critical than obturation density when claiming an RCT payment. This may explain why we had more cases with adequate filling length than those with complete obturation. In this study, both adequate filling length and complete obturation in the apical one-third of the root canal were used as the criteria for defining a GQEW. These criteria are similar to those for an acceptable root canal filling advocated by European Society of Endodontology (1994), i.e. a radiographically dense filling with its end located between 0 and $2 \mathrm{~mm}$ from the apex. By this definition, $34.8 \%$ of the treated root canals and $30.3 \%$ of the endodontically treated teeth fulfilled the criteria and, thus, qualified as having a GQEW. After adjustment, as a result of stratified sampling, the weighted average of root canals with GQEW was 30\% with an SE of 0.01 (data not shown) and that of teeth with GQEW was $25 \%$ with an SE of 0.02 (data not shown). These results indicate a disappointing level of technical quality of RCT in Taiwan. Similar findings of poor quality of RCT have also been reported by others (Petersson et al. 1986 (38\%), Ödesjö et al. 1990 (15.9\%), Imfeld 1991 (36\%), De Cleen et al. 1993 (49.4\%), Buckley \& Spångberg 1995 (42\%), Saunders et al. 1997 (38.6\%), Weiger et al. 1997 (14\%), Marques et al. 1998 (46\%), De Moor et al. 2000 (40.7\%), Kirkevang et al. 2000 (26.5\%), Boucher et al. 2002 (21\%)). However, Sidaravicius et al. (1999) reported an overall success rate of $65 \%$ for root-filled teeth.

The discrepancy in the results of the percentage of endodontically treated teeth with GQEW could be because of the use of different parameters to score the endodontic quality. Most of these studies adopted both adequate root filling length and proper obturation as the criteria for a GQEW (Petersson et al. 1986, Eckerbom et al. 1989, Ödesjö et al. 1990,Weiger et al. 1997, Kirkevang et al.2000, Boucher et al.2002). However, few other investigations used the level of the root filling as the only criterion for judging a GQEW (De Cleen et al. 1993, Saunders et al. 1997, De Moor et al. 2000). In addition, most of these studies considered the apical termination of the root filling $\leqq 2 \mathrm{~mm}$ from radiographic apex as the gold standard for an adequate root filling (Eckerbom et al. 1989, Ödesjö et al. 1990, Buckley \& Spångberg 1995, Boucher et al. 2002), whilst others used a broader standard of $\leqq 3 \mathrm{~mm}$ from the radiographic apex as a criterion for an adequate root filling (Marques et al. 1998, Kirkevang et al. 2001). In another study, only overfilling was considered as inadequate; the apical end of the root filling less than or greater than $2 \mathrm{~mm}$ from the radiographic apex was considered as adequate (Petersson et al. 1986). Theoretically, the broader the criteria, the better the 
results. According to the results of the previous studies, root-filled teeth with an adequate seal or filling length had a lower incidence of periapical lesions than teeth with an inadequate seal or filling length (Petersson et al. 1986, Eckerbom et al. 1989, Ödesjö et al. 1990, Kirkevang et al. 2001, Boucher et al. 2002).

In this study, the frequency of teeth with GQEW in anterior teeth $(40.4 \%)$ or in premolars $(33 \%)$ was significantly greater than that $(18.4 \%)$ in molars $(P<0.001)$. However, there was no significant difference in the frequency of teeth with GQEW between the anterior teeth and the premolars. In general, the anterior teeth or premolars have larger, straighter and fewer root canals than molars. Therefore, technically, an anterior tooth or a premolar is easier to treat than a molar, and a higher technical quality of RCT can be expected. Similar results of a higher success rate of RCT in anterior teeth or in premolars than in molars were also reported by others. Petersson et al. (1986) found that the percentage of teeth with complete obturation of the root canal without overfilling was $47 \%$ for premolars and $18 \%$ for molars. In addition, Boucher et al. (2002) showed that the frequency of teeth with acceptable filling was $34.2 \%$ for anterior teeth, $23.8 \%$ for premolars and $16.9 \%$ for molars.

Epidemiological investigations have revealed a significant difference in the results of endodontic treatment between well-trained endodontic specialists and general practitioners (Sjögren et al. 1990, Eriksen 1991, Friedman 1998). In this study, the cases were divided into hospital and private clinic, and further calculation and comparison were completed on the percentages of root canals or teeth with GQEW between these two groups. The percentage of root canals with GQEW in hospital cases was significantly greater $(43 \%)$ than that in private clinic cases $(28.8 \% ; P<0.001)$. Furthermore, the percentage of teeth with GQEW in hospital cases was also significantly greater $(38.1 \%)$ than that in private clinic cases (24.3\%; $P<0.001)$. In Taiwan, some teaching hospitals are affiliated with dental schools. There are well-established endodontic training programmes and welltrained endodontists in these teaching hospitals, where interns and residents work under the supervision of well-trained endodontic specialists. Therefore, endodontically treated cases completed by the dentists in the teaching hospitals can be expected to have a better technical quality than those completed by the dentists in the private clinics. However, the overall percentage of root canals or teeth with GQEW in Taiwan was still less than $35 \%$. This result indicates the need to improve the quality of endodontic treatment by the hospital dental workers and the general practitioners in Taiwan.

\section{Conclusions}

Approximately $70 \%$ of the teeth receiving RCT in Taiwan were either inadequately filled or incompletely obturated. These findings suggest that the technical standard of RCT is not satisfactory in Taiwan.

\section{Acknowledgements}

The authors extend their thanks to the Bureau of National Health Insurance in Taiwan for the help given during the study. The authors would also like to thank Dr Chun-Pin Chiang for many helpful suggestions. This study was supported by the grant DOH89-HI-1003 from the Department of Health, The Executive Yuan, Taiwan, Republic of China.

\section{References}

Boucher Y, Matossian L, Rilliard F, Machtou P (2002) Radiographic evaluation of the prevalence and technical quality of root canal treatment in a French subpopulation. International Endodontic Journal 35, 229-38.

Buckley M, Spångberg LSW (1995) The prevalence and technical quality of endodontic treatment in an American subpopulation. Oral Surgery, Oral Medicine, Oral Pathology, Oral Radiology and Endodontics 79, 92-100.

Cohen J (1960) A coefficient of agreement for nominal scales. Educational and Psychological Measurement 20, 37-46.

De Cleen MJH, Schuurs AHB,Wesselink PR,Wu MK (1993) Periapical status and prevalence of endodontic treatment in an adult Dutch population. International Endodontic Journal 26, 112-9.

De Moor RJG, Hommez GMG, De Boever JG, Delmé KIM, Martens GEI (2000) Periapical health related to the quality of root canal treatment in a Belgian population. International Endodontic Journal 33, 113-20.

Eckerbom M, AnderssonJE, Magnusson T (1987) Frequency and technical standard of endodontic treatment in a Swedish population. Endodontics and Dental Traumatology 3, 245-8.

Eckerbom M, Andersson JE, Magnusson T (1989) A longitudinal study of changes in frequency and technical standard of endodontic treatment in a Swedish population. Endodontics and Dental Traumatology 5, 27-31.

Eriksen HM (1991) Endodontology epidemiological considerations. Endodontics and Dental Traumatology 7, 189-95.

Friedman S (1998) Treatment outcome and prognosis of endodontic therapy. In: Orstavik D, Pitt Ford TR, eds. Essential Endodontology: Prevention and Treatment of Apical Periodontitis, 1st edn. Oxford, UK: Blackwell Science Ltd, 367-401.

Glantz SA (1997) Primer of Biostatistics, 4th edn. New York, USA: McGraw-Hill Incorporation, 137-40.

Hunt RJ (1986) Percent agreement, Person's correlation, and Kappa as measures of inter-examiner reliability. Journal of Dental Research 65, 128-30. 
Imfeld TN (1991) Prevalence and quality of endodontic treatment in an elderly urban population of Switzerland. Journal of Endodontics 17, 604-7.

Kirkevang LL, Hörsted-Bindslev P, Orstavik D, Wenzel A (2001) A comparison of the quality of root canal treatment in two Danish subpopulations examined 1974-75 and 1997-98. International Endodontic Journal 34, 607-12.

Kirkevang LL, Orstavik D, Hörsted-Bindslev P, Wenzel A (2000) Periapical status and quality of root filling and coronal restorations in a Danish population. International Endodontic Journal 33, 509-15.

Marques MD, Moreira B, Eriksen HM (1998) Prevalence of apical periodontitis and results of endodontic treatment in an adult, Portuguese population. International Endodontic Journal 31, $161-5$.

Ödesjö B, Helldén L, Salonen L, Langeland K (1990) Prevalence of previous endodontic treatment, technical standard and occurrence of periapical lesions in a randomly selected adult, general population. Endodontics and Dental Traumatology $\mathbf{6}$, 265-72.
Petersson K, Petersson A, Olsson B, Håkansson J, Wennberg A (1986) Technical quality of root fillings in an adult Swedish population. Endodontics and Dental Traumatology 2, 99-102.

Saunders WP, Saunders EM, Sadiq J, Cruickshank E (1997) Technical standard of root canal treatment in an adult Scottish sub-population. British Dental Journal 182, 382-6.

Sidaravicius B, Aleksejuniene J, Eriksen HM (1999) Endodontic treatment and prevalence of apical periodontitis in an adult population of Vilnius, Lithuania. Endodontics and Dental Traumatology 15, 210-5.

Sjögren U, Hägglund B, Sundqvist G, Wing K (1990) Factors affecting the long-term results of endodontic treatment. Journal of Endodontics 16, 498-504.

Valachovic RW, Douglass CW, Berkey CS, McNeil BJ, Chauncey HH (1986) Examiner reliability in dental radiography. Journal of Dental Research 65, 432-6.

Weiger R, Hitzer S, Hermle G, Löst C (1997) Periapical status, quality of root canal fillings and estimated endodontic treatment needs in an urban German population. Endodontics and Dental Traumatology 13, 69-74. 